\title{
Iterative Non-Rigid Image Registration in Brain MRI Images
}

\author{
Nasru Minallah', Muniba Ashfaq ${ }^{2}$ \\ ${ }^{1}$ Associate Professor, Department of Computer Systems Engineering, UET Peshawar KPK, Pakistan \\ 2Lecturer, Department of Computer Systems Engineering, UET Peshawar KPK, Pakistan
}

\section{A BSTRACT}

Non-rigid image registration plays a significant role in the medical imaging domain. It involves deformations of moving image pixels according to fixed or reference image anatomy. Accurate image registration is required for accurate medical diagnosis and clinical applications. Non-rigid image registration uses an optimization technique to find the best possible solution. The solution obtained using a single run of non-rigid image registration may be optimal but not much accurate. To increase accuracy in non-rigid image registration, multiple runs are required to improve the performance. Here in our research methodology, we used the Demon algorithm as non-rigid image registration for brain MRI images of a patient with a tumor. There is significant improvement i.e., an average of $66.61 \%$ decrease in the error of the results of iterative Demon algorithm as compared to non-iterative Demon algorithm

Keywords: Non-rigid Image Registration, Brain MRI, Iterative Non-rigid Image Registration, LBFGS, SSD

$\begin{array}{ll}\text { Author`s Contribution } & \text { Address of Correspondence } \\ \text { 1,2 Manuscript writing, Data Collection } & \text { Nasru Minallah } \\ \begin{array}{l}\text { Data analysis, interpretation, Conception, } \\ \text { synthesis, planning of research, and }\end{array} & \text { n.minallah@uetpeshwar.edu.pk } \\ \text { discussion } & \end{array}$

Cite this article: Minallah $N$, Ashfaq M. Iterative non-rigid image registration in Brain MRI Images. J. Inf. commun. technol. robot. appl.2020; 11(2):1-7

\section{Article info.}

Received: June 11, 2020

Accepted: December 14, 2020

Published: December 30, 2020

\section{INTRODUCTION}

Image registration is the process of finding optimal transformation so that two misaligned images are best aligned with each other. In the medical domain, image registration has a vital role in different aspects. There are two types of image registrations; rigid and non-rigid image registrations. In rigid image registration, different scans of the same patient do not involve any anatomical or pathological change (tumor growth) but there is a difference in patient positioning with respect to the imaging system [1]. The common parts of the human body that undergoes rigid image transformations are the head or skull and bone which is a rigid body. The patient's head may move during
Funding Source: Nil

Conflict of Interest: Nil scans or there may be geometrical differences between different acquisition systems [1]. On the other hand, non-rigid image registration is involved in those situations where there are local deformations. Non-rigid image registration is important in clinical follow-up studies [2-4]. This type of image registration is common in those parts of the human body that contain soft tissues and there are some deformations in them [5]. The deformations in soft tissues are due to the progression of disease e.g., tumor growth $[6,7]$ within the brain that affects not only normal brain structures but also there is volume expansion of lesion. Secondly, resection of a lesion in the brain after surgery affects 
the soft tissues of the brain. There is a difference in local deformations of soft tissues pre and post-surgical procedures. Moreover, non-rigid image registration is required where there is organ expansion or contraction e.g., lungs during respiration [8, 9]. It is also observed in cardiac motion between the scans [10-12], movement of fetus in mother's womb [13-16], dramatic growth of organs during fetal development [17], and moving kidneys [18]. The image registration that involves scans of the same subject is referred to as intra-subject image registration [19]. The second type involves image registration between two different subjects called inter-subject image registration [20]. It is used to analyze the morphological variability of a patient with respect to the population [21-23]. Non-rigid image registration can be used to segment different anatomical structures of the human body along with the presence of a lesion. This approach is called atlasbased image registration where not only the grayscale image but the corresponding labeled image is also available [24]. The grayscale image of the atlas when registered non-rigidly to the other patient may also segment the patient anatomy. The deformation field vector obtained from non-rigidly registering grayscale atlas image and patient image is further used to deform labeled image of atlas. In this way deformed labeled atlas image is used to segment the un-segmented patient scan by transferring the labels. This approach is more attractive in brain where lesion has intensity overlap with normal brain tissues and location map of normal brain structure using atlas is used to separate the lesion from healthy tissues. In order to successfully segment lesion as compared to healthy tissues, it is necessary to register the images accurately.

Non-rigid image registration techniques involve different deformation models. These deformation models depend on the regularization term that specifically identifies the type of physical model of deformation. Different deformation models can be used according to the specific medical application e.g., Elastic [25], Fluid [20], and Curvature [26]. The best non-rigid image registration result can be found using one of the optimization techniques. The optimization technique works on finding the best solution in terms of minimization of similarity measures between two images. In mono-modal image registration where both images are from the same domain SSD (Sum of Squared Differences) [27] is used in a wide variety of applications. In multi-modal image registration [28] where both images are from different modalities $\mathrm{Ml}$ (Mutual Information) [29] is used.

Technology advancements in non-rigid image registration techniques need to provide more accurate results. This research work is also related to providing more accurate results of non-rigid image registration techniques that have further diverse medical and clinical applications.

\section{LITERATURE REVIEW}

Non-rigid image registration has a significant role in medical imaging. To accurately register two images, there is a need of providing more accurate non-rigid image registration algorithms. Xiangbo Lin et al. [30] proposed Modified Active Demon (MAD) which improves in terms of accuracy and providing a smooth deformation field as compared to the Demon algorithm. Zhenchao Tang et al. [31] improved the active demon algorithm by introducing a new parameter balance coefficient to account for small and large deformations at the same time. They also work on improving registration accuracy and convergence speed and used a multi-level strategy in the registration approach. Sheng Lan et al. [32] introduce an image field to incorporate directional information in the image registration algorithm of Demons. Improved Demon algorithm is used in medical images [33] with the use of additional external force and gradient of mutual information with respect to the deformation field.

\section{METHODOLOGY}

The methodology used in our research project is the iterative procedure of applying non-rigid image registration. The iterative procedure depends on the selection of the maximum number of cycles for nonrigid image registration as shown in Fig 1. Demon algorithm is used as non-rigid image registration between template and reference image. In the first step template and the reference image are given as an input to the non-rigid image registration Demon algorithm. The solution found from this step is used to transform 
the template image. To further increase the accuracy of non-rigid image registration, this procedure is followed to the maximum number of cycles. The transformed template from the previous cycle is used in the next cycle to produce further accurate results.

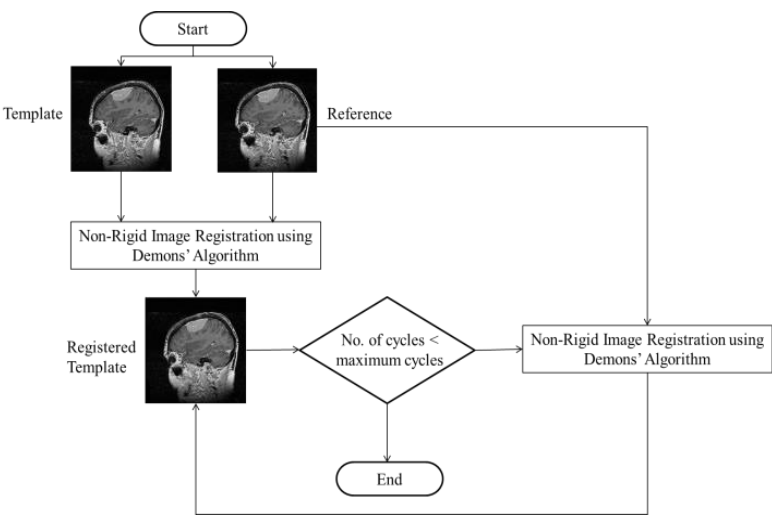

Figure 1. Workflow of Iterative Non-Rigid Image Registration using Demon Algorithm

\section{A. Demon Algorithm}

Demon algorithm is first introduced by Thirion [34] as non-rigid image registration. The demon forces are estimated by using the optical flow equation. In a static image $I_{1}$, for a given pixel point $p$, let $f_{1}$ be the intensity and $f_{2}$ be the intensity in a moving image $I_{2}$. The estimation of the displacement vector field $\mathbf{u}$ between the pixels $p$ and corresponding points in the image $I_{2}$.

$$
\mathbf{u}=\frac{\left(f_{2}-f_{1}\right) \nabla_{s}}{\|\nabla s\|^{2}+\left(f_{2}-f_{1}\right)^{2}}
$$

Where $\mathbf{u}=\left(u_{x}, u_{y}\right)$ is the estimation deformation field in $2 \mathrm{D} . \nabla s$ is the gradient in the static image $I_{1}$. Demon algorithm contains two forces internal and external. Internal force is edge-based as is represented here as $\nabla s$ an external force is represented here as $\left(f_{2}-f_{1}\right)$. To make the equation of velocity more stable, Thirion added $\left(f_{2}-f_{1}\right)$ the term. Gaussian smoothing is included as regularization as the deformation field is based on local information.

He Wang et al. [35] used edge forces of the moving image $I_{2}$ as well as compared to internal edge forces of the static image alone in the original equation. The additional forces further improve the non-rigid image registration performance in terms of speed and convergence.

$$
\mathbf{u}=\frac{\left(f_{2}-f_{1}\right) V s}{|\nabla s|^{2}+a^{2}\left(f_{2}-f_{1}\right)^{2}}+\frac{\left(f_{2}-f_{1}\right) V m}{|\nabla m|^{2}+a^{2}\left(f_{2}-f_{1}\right)^{2}}
$$

where ${ }^{\alpha}$ is the normalization factor to adjust the force-length and is proposed by Cachier et al. [36].

\section{B. Limited Memory BFGS}

Limited memory Broyden Fletcher Goldfarb Shanno (LBFGS) [37] is an optimization technique to find the optimal solution. LBFGS is less computational as compared to BFGS. It does not need to explicitly store the entire approximated Hessian matrix $H_{j}$. It is an iterative procedure and stores information from past $m$ iterations to implicitly approximate the inverse of the Hessian matrix.

The BFGS optimization technique on the other hand utilizes information from all the previous iterations for inverse Hessian computation. The first $m$ iterations of both LBFGS and BFGS produce the same search directions assuming initial search directions are the same. LBFGS is useful and successful in applications where the number of unknown parameters is large e.g., 3000 and even larger. LBFGS is successfully used as an optimization technique in the image registration paradigm where the solution lies at best-aligned images.

Table 1. Registration Error increases with Increasing Slice Distance

\begin{tabular}{|c|c|c|c|}
\hline $\begin{array}{c}\text { Slice } \\
\#\end{array}$ & $\begin{array}{c}\text { Initial } \\
\text { SSD }\end{array}$ & $\begin{array}{c}\text { SSD after Non-rigid } \\
\text { Image Registration }\end{array}$ & $\begin{array}{c}\text { Elapsed } \\
\text { Time (Sec) }\end{array}$ \\
\hline $\mathbf{4 9}$ & $1.34 \mathrm{E}+08$ & $5.23 \mathrm{E}+07$ & 73.048221 \\
\hline $\mathbf{4 8}$ & $1.24 \mathrm{E}+08$ & $4.96 \mathrm{E}+07$ & 44.734226 \\
\hline $\mathbf{4 7}$ & $1.10 \mathrm{E}+08$ & $4.36 \mathrm{E}+07$ & 58.975333 \\
\hline $\mathbf{4 6}$ & $9.30 \mathrm{E}+07$ & $3.85 \mathrm{E}+07$ & 79.502509 \\
\hline $\mathbf{4 5}$ & $6.31 \mathrm{E}+07$ & $3.19 \mathrm{E}+07$ \\
\hline $\mathbf{4 4}$ & \multicolumn{3}{|c|}{ Template Slice } \\
\hline $\mathbf{4 3}$ & $6.21 \mathrm{E}+07$ & $3.08 \mathrm{E}+07$ & 54.907187 \\
\hline $\mathbf{4 2}$ & $9.32 \mathrm{E}+07$ & $3.89 \mathrm{E}+07$ & 57.050865 \\
\hline $\mathbf{4 1}$ & $1.14 \mathrm{E}+08$ & $4.47 \mathrm{E}+07$ & 54.375758 \\
\hline $\mathbf{4 0}$ & $1.30 \mathrm{E}+08$ & $5.10 \mathrm{E}+07$ & 56.695653 \\
\hline $\mathbf{3 9}$ & $1.48 \mathrm{E}+08$ & $5.30 \mathrm{E}+07$ & 66.177859 \\
\hline \multicolumn{4}{|c|}{ Average Time } \\
\hline \multicolumn{3}{|c|}{}
\end{tabular}






Figure 2. Effect of increasing slice distance between reference and template on registration error

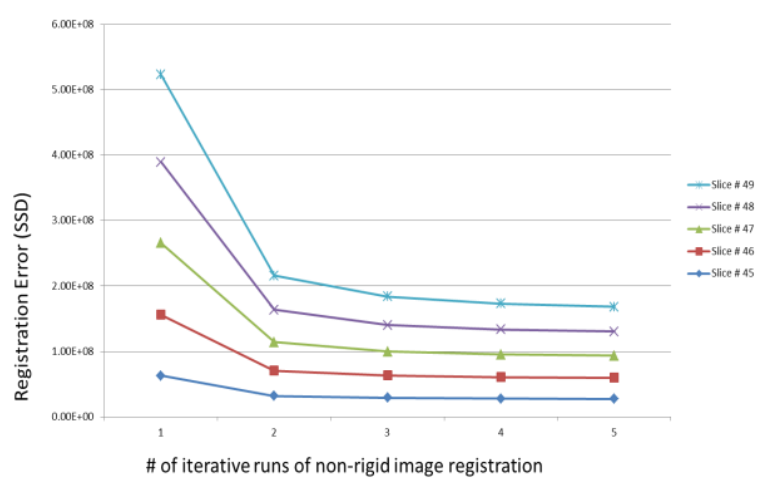

Figure 3. Effect of using iterative non-rigid image registration (Demon algorithm) on registration error

Table 2. Effect of an Increasing Number of Iteration of non-rigid image Registration on Image Registration Error

\begin{tabular}{|c|c|c|c|c|c|c|}
\hline Slice \# & Initial SSD & SSD after Run 1 & SSD after Run 2 & SSD after Run 3 & SSD after Run 4 & Percentage change \\
\hline $\mathbf{4 5}$ & $6.31 \mathrm{E}+07$ & $3.19 \mathrm{E}+07$ & $2.92 \mathrm{E}+07$ & $2.81 \mathrm{E}+07$ & $2.75 \mathrm{E}+07$ & -56.42 \\
\hline $\mathbf{4 6}$ & $9.30 \mathrm{E}+07$ & $3.85 \mathrm{E}+07$ & $3.42 \mathrm{E}+07$ & $3.28 \mathrm{E}+07$ & $3.22 \mathrm{E}+07$ & -65.38 \\
\hline $\mathbf{4 7}$ & $1.10 \mathrm{E}+08$ & $4.36 \mathrm{E}+07$ & $3.62 \mathrm{E}+07$ & $3.45 \mathrm{E}+07$ & $3.39 \mathrm{E}+07$ & -69.18 \\
\hline $\mathbf{4 8}$ & $1.24 \mathrm{E}+08$ & $4.96 \mathrm{E}+07$ & $4.07 \mathrm{E}+07$ & $3.80 \mathrm{E}+07$ & $3.66 \mathrm{E}+07$ & -70.48 \\
\hline 49 & $1.34 \mathrm{E}+08$ & $5.23 \mathrm{E}+07$ & $4.34 \mathrm{E}+07$ & $3.96 \mathrm{E}+07$ & $3.81 \mathrm{E}+07$ & -71.57 \\
\hline \multicolumn{5}{|c|}{ Average } & -66.61 \\
\hline
\end{tabular}

\section{EXPERIMENTAL RESULTS}

The research work is related to applying non-rigid image registration to brain MRI slices of the same patient in the same modality. This research project is divided into two phases. In the first phase, the effect of increasing slice distance on the similarity measure of images with reference to template slice is analyzed. Here, in this case, the template slice of the patient is constant and the reference or target image is changing. In the second phase, the effect of using iterative nonrigid image registration is analyzed with respect to the similarity measure of images. The dataset is the tumor base dataset of SPL (Surgical Planning Laboratory) of Harvard Medical School [38]. The dataset contains brain tumor MRI images of 10 patients. Patients are diagnosed with different types of tumors i.e., meningioma, low-grade glioma, and astrocytoma. The image resolution of these images is $256 \times 256 \times 124$. The total number of slices in each patient data is 124. In each patient, only one of the slices from the whole 3D MRI volume is selected. This selection of slice is based on the localization of the tumor and given to 4 experts for segmentation. The segmented slice doesn't only contain tumor but also brain, ventricles, and necrosis as other types. This dataset can be used for brain tumor segmentation. The manually segmented slice of a patient can further be used to segment the other nonsegmented slices of the brain. It is clear from the experiments shown in Table 1 that error increases as the distance between the slices increases. The graph in Fig 2. shows that there is a gradual increase in error as the target image is far away from the reference image. Here template image is slice no. 44 throughout the experiment and is constant. The reference image is changing; it is selected as 5 slices above and below the 
template image.

The template image is also called a moving image and the reference image is the target image and is fixed. The transformations in the template image change according to the reference image so that there is maximum similarity. The similarity measure used here is SSD (Sum of Squared Difference). The solution lies in the minimization of SSD in an optimization technique. The optimization technique used here is LBFGS that works on the minimization of SSD. The initial SSD is the similarity measure before applying for demon registration and after applying the demon algorithm SSD is reduced. This shows that after applying for non-rigid image registration, the template image is now much close to the reference or target image. The initial and final SSD of one slice above and below from the template image is almost the same. Similar is the case for other slices with the same distance above and below of the template slice. There is another set of experiments in which the result of nonrigid image registration undergoes another cycle of nonrigid image registration. Similarly, a total of four cycles of non-rigid image registration is applied. The error between the template and target image is further reduces in the second, third, and fourth cycles of nonrigid image registration. The results are shown in Table 2 that applying the demon algorithm iteratively can significantly reduce the error between template and reference image. The corresponding elapsed time is also shown in Table 3. There is significant performance improvement in iterative non-rigid image registration as compared to non-iterative non-rigid image registration as shown in Fig 3. The elapsed time is increased in iterative non-rigid image registration as compared to non-iterative non-rigid image registration.

Table 3. Elapsed Time (sec) of each run of Non-Rigid Image Registration

\begin{tabular}{|c|c|c|c|c|c|}
\hline Slice \# & Elapsed Time 1 $\mathbf{( s e c )}$ & $\begin{array}{c}\text { Elapsed Time 2 } \\
(\mathbf{s e c})\end{array}$ & $\begin{array}{c}\text { Elapsed Time 3 } \\
(\mathbf{s e c})\end{array}$ & $\begin{array}{c}\text { Elapsed Time 4 } \\
(\mathbf{s e c})\end{array}$ & Total Time (sec) \\
\hline $\mathbf{4 5}$ & 60.947201 & 35.924276 & 28.693966 & 24.972769 & 150.538212 \\
\hline $\mathbf{4 6}$ & 78.218659 & 27.433607 & 27.773548 & 30.449648 & 163.875462 \\
\hline $\mathbf{4 7}$ & 67.956193 & 67.789047 & 47.129103 & 41.437823 & 224.312166 \\
\hline $\mathbf{4 8}$ & 57.976589 & 53.702601 & 39.667511 & 37.543219 & 188.88992 \\
\hline $\mathbf{4 9}$ & 80.515352 & 84.271723 & 58.072798 & 42.683631 & 265.543504 \\
\hline
\end{tabular}

\section{DISCUSSION}

Non-rigid image registration in medical images is required where there is a local difference between the pixels of the template image and the reference image. The pixels in the template image move independently of each other and according to the reference image. The optimization technique iteratively tries to find the best solution in terms of minimization of similarity measure between template and reference image. The distance between template and reference slice is directly proportional to the difference between the similarities of the two. The high similarity between template and reference images shows more aligned images locally with reduced error. In our research methodology, registered template image after one complete run of non-rigid image registration undergoes another three more cycles of non-rigid image registration. Whole 3D volume using non-rigid image registration. The increasing distance of the reference slice from the template slice increases the error between the two. The non-rigid image registration technique performs well in the situation where the similarity increase as the number of complete runs of non-rigid image registration increase. The performance in terms of reduced error increases with an increase in computational time.

\section{CONCLUSION}

Non-rigid image registration has significant importance in the medical domain. There are anatomical changes in the soft tissues due to resection or progression of brain tumors. To analyze the change in the tumor growth or after a surgical procedure of tumor excision; non-rigid image registration techniques 
are required to deform previous visit scans with respect to next visit scans. The segmented previous scan of the patient can further be used to segment the next visit scans. Moreover, a single segmented slice of the patient can be used to segment the initial error between two images is less. The increased error due to the increasing distance between the template and reference slice can significantly be reduced by using iterative cycles of non-rigid image registration.

\section{REFERENCES}

1. Hill, D.L., et al., Medical image registration. 2001. 46(3): p. R1.

2. Aljabar, P., et al. Analysis of growth in the developing brain using non-rigid registration. in 3rd IEEE International Symposium on Biomedical Imaging: Nano to Macro, 2006. 2006. IEEE.

3. Cachier, P. and D. Rey. Symmetrization of the non-rigid registration problem using inversion-invariant energies: Application to multiple sclerosis. in International Conference on Medical Image Computing and Computer-Assisted Intervention. 2000. Springer.

4. Rey, D., et al., Automatic detection and segmentation of evolving processes in 3D medical images: Application to multiple sclerosis. 2002. 6(2): p. 163-179.

5. Zeng, Q., et al. Weakly non-rigid MR-TRUS prostate registration using fully convolutional and recurrent neural networks. in Medical Imaging 2020: Image Processing. 2020. International Society for Optics and Photonics.

6. Bauer, S., L.-P. Nolte, and M. Reyes. Segmentation of brain tumor images based on atlas-registration combined with a Markov-Random-Field lesion growth model. in 2011 IEEE International Symposium on Biomedical Imaging: From Nano to Macro. 2011. IEEE.

7. Zacharaki, E.I., et al., Non-diffeomorphic registration of brain tumor images by simulating tissue loss and tumor growth. 2009. 46(3): p. 762-774.

8. Haghighi, B., et al., A GPU-based symmetric non-rigid image registration method in human lung. 2018. 56(3): p. 355-371.

9. Weick, S., et al., Non-rigid image registration of 4D-MRI data for improved delineation of moving tumors. 2020. 20: p. 1-10.

10. Heyde, B., et al. Three-dimensional cardiac motion estimation based on non-rigid image registration using a novel transformation model adapted to the heart. in International Workshop on Statistical Atlases and Computational Models of the Heart. 2012. Springer.

11. Myronenko, A., X. Song, and D.J. Sahn. Maximum likelihood motion estimation in 3D echocardiography through non-rigid registration in spherical coordinates. in International Conference on Functional Imaging and Modeling of the Heart. 2009. Springer.
12. Li, B., A.A. Young, and B.R. Cowan. GPU accelerated non-rigid registration for the evaluation of cardiac function. in International Conference on Medical Image Computing and Computer-Assisted Intervention. 2008. Springer.

13. Woo, J., et al., Non-rigid ultrasound image registration based on intensity and local phase information. 2009. 54(1-3): p. 33-43.

14. Kuklisova-Murgasova, M., et al., Distortion correction in fetal EPI using non-rigid registration with a Laplacian constraint. 2017. 37(1): p. 12-19.

15. You, W. and C.J.b. Limperopoulos, Inter-slice motion correction using spatiotemporal interpolation for functional magnetic resonance imaging of the moving fetus. 2017: p. 204404.

16. Parraguez, S.P., Fast and robust methods for non-rigid registration of medical images. 2014, Imperial College London.

17. Rueckert, D. and P.J.I.S.P.M. Aljabar, Nonrigid registration of medical images: Theory, methods, and applications [applications corner]. 2010. 27(4): p. 113119.

18. Zöllner, F.G., et al., Assessment of 3D DCE-MRI of the kidneys using non-rigid image registration and segmentation of voxel time courses. 2009. 33(3): p. 171181.

19. Wei, D., et al., SLIR: Synthesis, localization, inpainting, and registration for image-guided thermal ablation of liver tumors. 2020. 65: p. 101763.

20. D'agostino, E., et al., A viscous fluid model for multimodal non-rigid image registration using mutual information. 2003. 7(4): p. 565-575.

21. Bai, J., et al., Atlas-based automatic mouse brain image segmentation revisited: model complexity vs. image registration. 2012. 30(6): p. 789-798.

22. Ahmad, E., et al. Atlas-registration based image segmentation of MRI human thigh muscles in 3D space. in Medical Imaging 2014: Image Perception, Observer Performance, and Technology Assessment. 2014. International Society for Optics and Photonics.

23. Wang, Y. and L.H.J.M.i.a. Staib, Physical model-based non-rigid registration incorporating statistical shape information. 2000. 4(1): p. 7-20.

24. Scheys, L., et al., Atlas-based non-rigid image registration to automatically define line-of-action muscle models: a validation study. 2009. 42(5): p. 565-572.

25. Kybic, J. and M.J.I.t.o.i.p. Unser, Fast parametric elastic image registration. 2003. 12(11): p. 1427-1442.

26. Fischer, B., J.J.J.o.M.I. Modersitzki, and Vision, Curvature based image registration. 2003. 18(1): p. 8185.

27. Prabhu, R. and A. Khan, A study of biomedical image registration algorithms. 2017.

28. So, R.W. and A.C. Chung. Multi-modal non-rigid image registration based on similarity and dissimilarity with the prior joint intensity distributions. in 2010 IEEE 
International Symposium on Biomedical Imaging: From Nano to Macro. 2010. IEEE.

29. Gaens, T., et al. Non-rigid multimodal image registration using mutual information. in International Conference on Medical Image Computing and Computer-Assisted Intervention. 1998. Springer.

30. Lin, X., et al. An improved method of "Demons" non-rigid image registration algorithm. in 2008 9th International Conference on Signal Processing. 2008. IEEE.

31. Tang, Z., et al. An effective non-rigid image registration method based on active demons algorithm. in 2016 IEEE 29th International Symposium on Computer-Based Medical Systems (CBMS). 2016. IEEE.

32. Lan, S., Z. Guo, and J.J.P.R.L. You, Non-rigid medical image registration using image field in Demons algorithm. 2019. 125: p. 98-104.

33. Zhang, H., W. ZHANGJ, and J.J.O.P.E. SUN, Non-rigid medical image registration based on improved Demons algorithm. 2007. 15(1): p. 1452150.

34. Thirion, J.-P., Image matching as a diffusion process: an analogy with Maxwell's demons. 1998.

35. Wang, H., et al., Validation of an accelerated 'demons' algorithm for deformable image registration in radiation therapy. 2005. 50(12): p. 2887.

36. Cachier, P., X. Pennec, and N. Ayache, Fast non rigid matching by gradient descent: Study and improvements of the" demons" algorithm. 1999.

37. Chen, K., et al., Improved optimization methods for image registration problems. 2019. 80(2): p. 305-336.

38. https://spl.harvard.edu/software-and-data-sets 